\title{
Pheochromocytoma with Catecholamine-Induced Cardiomyopathy: Not an Appropriate Case for Laparoscopic Procedure
}

\author{
Anuj Jain, Surendra Singh
}

\begin{abstract}
Laparoscopic surgery results in multiple postoperative benefits, allowing for quicker recovery and shorter hospital stay, but this is not a panacea. Catecholamine-induced cardiomyopathy $(\mathrm{CIC})$ is well known in patients with persistently elevated catecholamine levels in the blood. The complex interaction between existing cardiac pathology and laparoscopy associated cardiovascular changes may precipitate heart failure in patients with limited cardiac reserve. We report a diagnosed case of adrenal pheochromocytoma undergoing laparoscopic adrenalectomy. A 2D echocardiography done at the time of presentation demonstrated dilated left ventricle (LV) with global hypokinesia and ejection fraction (EF) of $25 \%$ with moderate mitral regurgitation (MR). The patient received phenoxybenzamine, 20 mg TDS; propanolol, $10 \mathrm{mg}$ TDS; ramipril, $2.5 \mathrm{mg}$ OD and Amlodipine, $5 \mathrm{mg} \mathrm{BD}$ for medical optimization prior to excision of pheochromocytoma. Echocardiography after the start of medical therapy now showed a normal sized LV with EF of $40 \%$ with no regional wall motion abnormality with mild MR. Pneumoperitoneum was created with carbon dioxide. Subsequent to peritoneal insufflation patient developed features of left ventricular failure. The laparoscopy was abandoned and laparotomy was done. The surgery could proceed successfully without major intraoperative and postoperative problems.
\end{abstract}

Keywords: Pheochromocytoma, Left ventricular failure, Catecholamine-induced cardiomyopathy.

How to cite this article: Jain A, Singh S. Pheochromocytoma with Catecholamine-Induced Cardiomyopathy: Not an Appropriate Case for Laparoscopic Procedure. World J Endoc Surg 2012;4(3):105-107.

\section{Source of support: Nil}

Conflict of interest: None

\section{INTRODUCTION}

Laparoscopic surgery results in multiple postoperative benefits, allowing for quicker recovery and shorter hospital stay. These advantages are the reason for growing acceptance of laparoscopic method proposed for many surgical procedures. This communication does not refute the advantages of laparoscopy, but emphasizes that this is not a panacea. The physiological changes which occur during laparoscopy may not be well-tolerated by a certain group of patients.

Catecholamine-induced cardiomyopathy (CIC) is well known in patients with persistently elevated catecholamine levels in the blood. Catecholamines and their oxidative products cause a direct toxic effect on the myocardium. Clinical features of CIC include hypertension, dilated or hypertrophic cardiomyopathy, pulmonary edema due to cardiogenic or noncardiogenic factors, cardiac arrhythmia and cardiac arrest. The definitive management of CIC include medical treatment with $\alpha$-blockers, angiotensin converting enzyme inhibitors (ACEIs) and $\beta$-adrenergic blockers followed by excision of catecholamine-producing tumor. ${ }^{1}$ The myocardium in these patients is less sensitive to catecholamine infusions which may be important for postoperative management of these patients after excision of the catecholamine-producing tumor.

The complex interaction between existing cardiac pathology and laparoscopy associated cardiovascular changes may precipitate heart failure in patients with limited cardiac reserve.

\section{CASE REPORT}

We report a diagnosed case of adrenal pheochromocytoma undergoing laparoscopic adrenalectomy.

The patient was a 23 years female weighing merely $30 \mathrm{~kg}$ with clinical features suggestive of pheochromocytoma. Two months prior to surgery patient developed dyspnea on exertion NYHA grade III along with pitting pedal edema. A 2D echocardiography was done which demonstrated dilated left ventricle (LV) with global hypokinesia and ejection fraction (EF) of 25\% with moderate mitral regurgitation (MR). Definitive diagnosis of pheochromocytoma was made after metanephrines were found raised in the urine and serum.

The patient received phenoxybenzamine, $20 \mathrm{mg}$ TDS; propanolol, $10 \mathrm{mg}$ TDS; ramipril, $2.5 \mathrm{mg}$ OD; and amlodipine, $5 \mathrm{mg}$ BD for medical optimization prior to excision of pheochromocytoma. Patient showed significant improvement in the clinical signs and symptoms after the start of the medical therapy. Echocardiography after the start of medical therapy now showed a normal sized LV with EF of $40 \%$ with no regional wall motion abnormality with mild MR.

Patient was scheduled for laparoscopic adrenalectomy under general anesthesia (GA). Tablet lorazepam $1 \mathrm{mg}$ was given as premedication. Antihypertensive medications were continued as per their schedule. 
Monitoring included a 5-lead electrocardiogram (EKG) with arrhythmia analysis and ST segment monitoring, pulse oximetry, invasive blood pressure and central venous pressure (CVP). Induction of anesthesia was done with fentanyl, $100 \mu \mathrm{g}$; midazolam, $1 \mathrm{mg}$; lignocaine, $60 \mathrm{mg}$; thiopentone, $75 \mathrm{mg}$; vecuronium, $3 \mathrm{mg}$. Endotracheal intubation was performed and anesthesia was maintained using air oxygen mixture with sevoflurane $2 \%$. The patient was hemodynamically stable during induction of GA and after tracheal intubation. Pneumoperitoneum was created with carbon dioxide. Intra-abdominal pressure was limited to $10 \mathrm{~mm} \mathrm{Hg}$ with gas insufflation rate at $2 \mathrm{l} / \mathrm{min}$. Subsequent to peritoneal insufflation there was a sudden rise in the diastolic blood pressure (DBP) and CVP followed by a fall in the systolic blood pressure (SBP) albeit not alarming but SBP showed a falling trend while heart rate (HR), CVP, DBP were slowly rising. A normal sinus rhythm was maintained. A few minutes later the oxygen saturation $\left(\mathrm{SpO}_{2}\right)$ started showing a downward trend gradually decreasing to $92 \%$ at which time inspired oxygen concentration was increased to $60 \%$ and later to $70 \%$. The airway pressures (both $\mathrm{P}_{\text {peak }}$ and $\mathrm{P}_{\text {plateau }}$ ) were also showing a persistent increasing trend (Fig. 1). Auscultation of both the hemithoraces was done to rule out endobronchial migration of the endotracheal tube (ETT). After confirming proper tube positioning, adequate muscle relaxation, adequate depth of anesthesia and excluding fluid overload, a correlation of all the events was done which concluded to the provisional diagnosis of intraoperative left ventricular failure (LVF) secondary to increased cardiac afterload due to pneumoperitoneum.

The surgeon was informed about the events and was suggested to convert to open procedure. The laparoscopy was abandoned and laparotomy was done. As soon as the pneumoperitoneum was removed (indicated by arrow in the Fig. 1) there was an improvement in the CVP, DBP and airway pressure with more gradual improvement in the SBP, $\mathrm{HR}$ and $\mathrm{SpO}_{2}$.

The surgery could proceed uneventfully apart from the usual hemodynamic changes anticipated during excision of pheochromocytoma. Interpleural catheter was inserted for analgesia and the patient was electively ventilated for 24 hours postoperatively. She was weaned out of the ventilator without major problem.

\section{DISCUSSION}

Pathophysiology of the heart due to CIC must be understood clearly in order to appreciate the importance of the intraoperative events. Cardiac physiology can be grossly described in terms of five parameters, i.e. preload, afterload, contractility, heart rate and rhythm. A persistently raised levels of catecholamines induce a cardiomyopathy severely affecting the contractility of the myocardium; at the same time catecholamines also induce intense vasoconstriction raising the afterload of the left ventricle (LV). So the LV persistently works against an increased resistance and when the ongoing myocardial insult in severe enough, LV failure sets in manifesting as dyspnea, edema.

CIC also leads to dysfunction of the papillary muscle apparatus leading to onset of MR. MR leads to chronic volume overload leading to dilatation of the LV cavity. As dilatation of the $\mathrm{LV}$ occurs there is increased tension on the myocardium thereby increasing the myocardial oxygen demand leading to further aggravation of the clinical scenario.

The only modifiable factor in this case was the LV afterload. After starting the phenoxybenzamine (an $\alpha$ blocker), ramipril (ACEIs) and amlodepine (calcium channel blocker) the afterload of the heart was decreased thereby improving cardiac output (CO) resulting in improvement of the clinical features. But it is important to understand that this improvement in the clinical profile was not because of the improvement of the contractility of the heart but was due to extremely low LV afterload due to the antihypertensive drugs that the patient was receiving.

Pneumoperitoneum during laparoscopy leads to increased cardiac afterload via several mechanisms. Stretching of peritoneum leads to increased sympathetic stimulation ${ }^{2}$ resulting in release of catecholamine and vasopressin and a further increase in afterload. This sympathetic stimulation due to peritoneal stretching may be abnormally high in patients with pheochromocytoma. Increased afterload also increases the regurgitant fraction

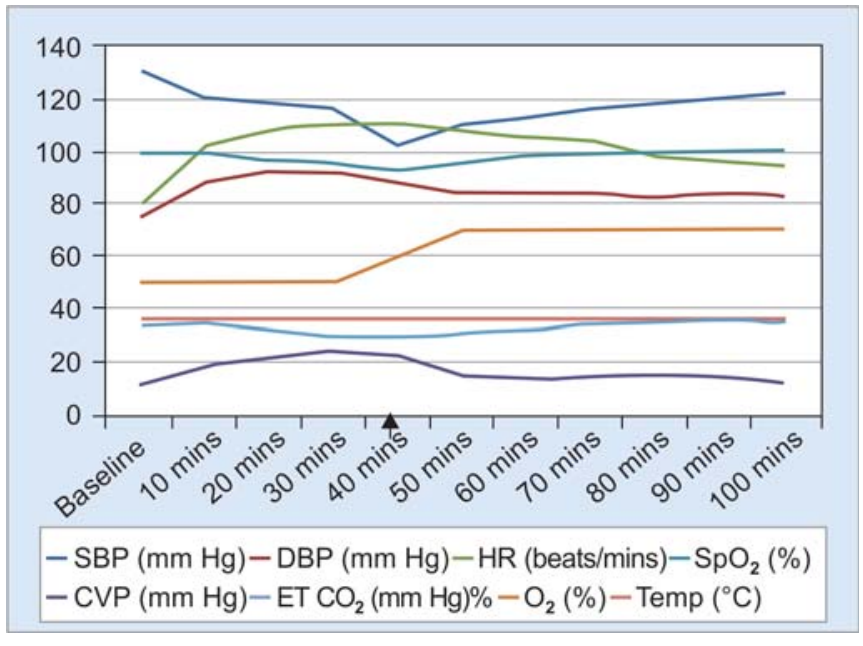

Fig. 1: Trend of hemodynamic parameters monitored from the start of induction of anesthesia up to 100 minutes after removal of pneumoperitoneum. The arrow represents the time at which pneumoperitoneum was removed 
of MR leading to an increase in left ventricular end diastolic pressure (LVEDP) and inturn increase the pulmonary venous pressure leading to pulmonary edema. The increase in afterload is well tolerated by normal patients but can cause severe decrease in cardiac output in patients with preexisting cardiac disease. $^{3}$

Another important factor precipitating LV failure (LVF) was the fluid shifts toward the heart due to inferior vena cava (IVC) compression caused by increased IAP..${ }^{4,5}$ Hence, increased MR, increased afterload and compromised LV contractility all contributed to precipitation of LVF characterized by decreasing pulse pressure, increased heart rate, drop in saturation and an increase in airway pressure in this case.

This case report is important because at first instance there were no obvious contraindications to a laparoscopy once the patient had been optimized on antihypertensive drugs. Laparoscopic technique is well established for excision of pheochromocytoma. Laparoscopic excision of pheochromocytoma can be done in patients with isolated moderate systolic dysfunction and also in patients with isolated moderate MR. When both these abnormalities are present in the patient at the same time, which may be a common presentation considering the pathophysiology of CIC, then laparoscopy may not be tolerated and may become life-threatening. A preoperative $\alpha$-blockade therapy may decrease the afterload of the left ventricle to such an extent that it may mask the clinical features of compromised LV function. Although medical management could have been tried in this case but considering the duration of surgery this would have been inappropriate and best possible alternative would have been conversion to an open approach. This case could be managed without an apparent lifethreatening event because of early anticipation of events and termination of laparoscopy. An open procedure under general anesthesia with thoracic epidural would be the ideal choice in similar patients.

\section{CONCLUSION}

Increased care is to be taken while selecting patients for laparoscopic excision of pheochromocytoma. Presence of a preoperative history of congestive heart failure, dilated left ventricle and moderate to severe MR may be at high risk for developing LVF during laparoscopy.

\section{REFERENCES}

1. Kassim TA, et al. Catecholamine induced cardiomyopathy. Endocr Pract 2008 Dec;19(9):1137-49.

2. Wahba RW, Beique F, Kleiman SJ. Cardiopulmonary function and laparoscopic cholecystectomy. Can J Anaesth 1995;42:51.

3. Harris SN, Ballantyne GH, Luther MA, et al. Alterations of cardiovascular performance during laparoscopic colectomy: A combined hemodynamic and echocardiographic analysis. Anesth Analg 1996;83:482.

4 Kashtan J, Green JF, Parsons EQ, et al. Hemodynamic effect of increased abdominal pressure. J Surg Res 1981;30:249.

5. Giebler RM, Behrends M, Steffens T, et al. Intraperitoneal and retroperitoneal carbon dioxide insufflation evoke different effects on caval vein pressure gradients in humans: Evidence for the starling resistor concept of abdominal venous return. Anesthesiology 2000;92:1568.

\section{ABOUT THE AUTHORS}

\section{Anuj Jain (Corresponding Author)}

Senior Resident, Department of Anesthesiology, Sanjay Gandhi Postgraduate Institute of Medical Sciences, Lucknow, Uttar Pradesh India, e-mail: anuj.jain.mln@gmail.com

\section{Surendra Singh}

Professor, Department of Anesthesiology, Sanjay Gandhi Postgraduate Institute of Medical Sciences, Lucknow, Uttar Pradesh, India 